\title{
Quantitative videocapillaroscopy correlates with functional respiratory parameters: a clue for vasculopathy as a pathogenic mechanism for lung injury in systemic sclerosis
}

\author{
Alfredo Guillén-Del-Castillo ${ }^{1}$, Carmen Pilar Simeón-Aznar ${ }^{1 *}$, Eduardo L. Callejas-Moraga ${ }^{2}$, Carles Tolosa-Vilella², \\ Serafín Alonso-Vila', Vicente Fonollosa-Pla ${ }^{1}$ and Albert Selva-O'Callaghan ${ }^{1}$
}

\begin{abstract}
Background: To determine whether lung involvement is related to microvascular perturbations, nailfold videocapillaroscopy (NVC) was performed in patients with systemic sclerosis (SSC).

Methods: A cross-sectional study was consecutively accomplished in 152 SSc patients. NVC, a pulmonary function test and echocardiography were undergone within a 3-month period. Finally, 134 patients with at least eight NVC (200x magnification) images were selected for quantitative and qualitative examinations.

Results: Patients with interstitial lung disease presented lower median capillary density $(4.86 / \mathrm{mm}$ vs $5.88 / \mathrm{mm}, p=0.005)$ and higher median of neoangiogenesis $(0.56 / \mathrm{mm}$ vs $0.31 / \mathrm{mm}, p=0.005)$. A higher quantity of neoangiogenesis capillaries was found in patients with pulmonary arterial hypertension $(0.70 / \mathrm{mm}$ vs $0.33 / \mathrm{mm}, p=0.008)$. Multivariate linear regression analysis established a correlation between neoangiogenesis and decreased forced vital capacity (FVC) $(p<0.001)$ : for each capillary with neoangiogenesis visualized on average per $1 \mathrm{~mm}$, FVC was $7.3 \%$ reduced. In qualitative NVC, a late pattern as defined by Cutolo was also associated with lower FVC $(p=0.018)$. The number of giant capillaries was associated with reduced diffusion capacity of the lung for carbon monoxide (DLCO) $(p=0.016)$; for each giant capillary per $1 \mathrm{~mm}$, DLCO was $11.8 \%$ diminished.
\end{abstract}

Conclusions: A good correlation was observed between distinctive quantitative and qualitative NVC features with lung functional parameters such as FVC and DLCO. It is suggested that vasculopathy could play a role in SSc lung involvement.

Keywords: Interstitial lung disease, Nailfold videocapillaroscopy, Pulmonary hypertension, Scleroderma, Systemic sclerosis

\section{Background}

Systemic sclerosis (SSc) is a connective tissue disease characterized by microvascular damage. It seems that endothelial activation induces the release of inflammatory mediators, platelet activation and inflammatory cell recruitment [1-3]. Subsequently, intimal and smooth muscle vessel wall proliferation ensues, causing luminal

\footnotetext{
* Correspondence: cpsimeon@vhebron.net

${ }^{1}$ Department of Systemic Autoimmune Diseases, Hospital Universitari Vall d'Hebron, Universitat Autònoma de Barcelona, Passeig Vall d'Hebron 119129, 08035 Barcelona, Spain

Full list of author information is available at the end of the article
}

obstruction and activation of myofibroblasts that results in architectural alterations due to an excessive extracellular matrix deposition, reduction in the number of capillaries, tissue hypoxia and dysfunction of vascular regeneration [4].

The lung is one of the most frequently jeopardized organs in SSc; thus interstitial lung disease (ILD) affects around $55-65 \%$ of patients subjected to high-resolution computed tomography (HRCT) [5, 6], and pulmonary arterial hypertension (PAH) diagnosed by right heart catheterization (RHC) involves approximately $10 \%$ of this population $[7,8]$. As a result of its high prevalence 
and poor prognosis, pulmonary involvement is nowadays the major cause of SSc-related deaths [9-11]. An exhaustive study of the relation between capillaroscopy features and lung manifestations would facilitate comprehension of the pulmonary pathophysiology.

Capillary microscopy was initially used as a qualitative technique to describe microvascular abnormalities in SSc and other autoimmune-related diseases. Maricq et al. [12] defined the scleroderma pattern as a group of capillaroscopy findings with potential diagnostic value in this disease. Later, these were detailed into active and slow capillaroscopy patterns [13]. More recently, using nailfold videocapillaroscopy (NVC), Cutolo et al. [14] defined a new classification of qualitative capillaroscopy abnormalities into early, active and late scleroderma patterns. The late pattern was related to lower levels of circulating endothelial progenitor cells and higher vascular endothelial growth factor (VEGF), demonstrating that an impairment of vasculogenesis mediates the loss of capillaries [15]. Recently, a sequential quantitative NVC study relating the presence of new incident capillaroscopy features to disease progression has been reported [16]. Nevertheless, no prior studies were focused on extensive quantitative NVC analysis and its correlation with objective lung parameters.

The hypothesis of the present study was that microcirculation damage leads the pathogenesis of the main manifestations in SSc such as ILD and PAH. The main aim of the work was to analyse NVC alterations using a quantitative method and to investigate their correlation with the pulmonary function test (PFT).

\section{Methods}

\section{Patients}

A cross-sectional study was carried out on SSc patients from the Vall d'Hebron Hospital cohort. All patients fulfilled LeRoy et al.'s SSc classification criteria [17], and accomplishment of the 2013 ACR/EULAR classification criteria was also assessed [18]. The study was approved by the Ethics Committee for Clinical Research (PG(AG)4/2015), and all patients provided written informed consent for their participation. NVC was consecutively conducted in 152 patients over a 12-month period. PFTs and echocardiography were performed within a 3-month period. Thirteen patients were excluded with fewer than eight explored nailfold fields, and another five patients for being lung transplant recipients. Finally, 134 SSc patients were selected for the analyses. The disease onset was described as the date of first symptom attributable to SSc including Raynaud's phenomenon (RP). All clinical or parameter data were collected at the time of NVC. Study quality was assessed by the Strengthening the Reporting of Observational
Studies in Epidemiology (STROBE) checklist for cross-sectional studies [19].

\section{Clinical manifestations}

Cutaneous subsets were defined as previously [20], according to the extent of skin thickening: limited cutaneous SSc (lcSSc), if skin sclerosis was distal to the elbows and knees or the face; diffuse cutaneous SSc (dcSSc), if skin sclerosis was extended proximally to the elbows or knees; and sine scleroderma SSc (ssSSc), if there was no skin involvement. Manifestations collected included RP, telangiectasias, past or current digital ulcers (DU), calcinosis and past history of scleroderma renal crisis (SRC).

Interstitial lung disease was defined as radiologic evidence of interstitial findings on HRCT examined by an expert thorax radiologist [21]. PAH was defined as mean pulmonary arterial pressure (mPAP) $\geq 25 \mathrm{mmHg}$ with pulmonary artery wedge pressure (PAWP) $\leq 15 \mathrm{mmHg}$, and pulmonary vascular resistance $(\mathrm{PVR})>3$ Wood units in right heart catheterization (RHC) [22, 23]. Cardiac involvement was defined as past or current pericardial effusion, left ventricular ejection fraction (LVEF) < $50 \%$, macrovascular or microvascular ischemic heart disease with no cardiovascular risk factor (CVRF), conduction abnormalities, diastolic dysfunction with no CVRF or mitral regurgitation with no CVRF [24].

Musculoskeletal disease included arthritis, tendon fiction rubs, joint contractures and myositis. Gastrointestinal SSc disease was established if any of the following were present: oesophageal dysmotility, gastric antral vascular ectasia, gastric or bowel dysmotility, intestinal bacterial overgrowth, intestinal pseudo-obstruction or anal sphincter dysfunction.

\section{Immunology features}

Antinuclear antibodies (ANA) were evaluated by indirect immunofluorescence (IIF) assay using HEp-cell line 2. Anticentromere antibodies (ACA) were described by IIF, and anti-topoisomerase I antibodies were determined by enzyme-linked immunosorbent assay. A commercial line blot assay was performed to detect anticentromere proteins $\mathrm{A}$ and $\mathrm{B}$, anti-RNA pol III and anti-PM/Scl antibodies (EUROLINE Systemic Sclerosis (Nucleoli) Profile (IgG), Euroimmun, Germany) according to the manufacturer's instructions.

\section{Pulmonary function test and echocardiography}

Complete PFTs including the percentage of the predicted forced vital capacity (FVC) and the percentage of the predicted diffusion capacity of the lung for carbon monoxide (DLCO) were accomplished using MasterLab equipment (MasterLab, Jaegger, Germany), following the ATS/ERS recommendations [25]. Echocardiography was performed by an experienced echocardiographer using a 
Vivid E9 system (General Electric Vingmed, Horten, Norway), according to the consensus guidelines [26]. Right ventricular systolic pressure (RVSP), tricuspid regurgitation velocity (TRV) and the presence of other echo pulmonary hypertension $(\mathrm{PH})$ signs were measured. In order to avoid loss of data information about patients with no measurable TRV, the variable 'TRV $\geq$ $2.9 \mathrm{~m} / \mathrm{s}$ or other echo $\mathrm{PH}$ signs' was created [23].

\section{Nailfold videocapillaroscopy}

Nailfolds were examined using an Optilia Digital Videocapillaroscope (Optilia Instruments AB, Sollentuna, Sweden) with $200 \times$ lens magnification and an LED lamp by the same operator (AS-O) who was blinded to clinical patients' conditions. Each patient was acclimatized for at least $20 \mathrm{~min}$ at room temperature of $20-24^{\circ} \mathrm{C}$ before the examination. NVC was conducted with a contact adapter and immersion oil dropper, studying the middle nailfold from the second to fifth fingers of both hands. Optipix Lite software (Optilia Instruments $A B$ ) was used for visualization of consecutive 1-mm-wide images of first-line capillaries. Two images were taken on each finger, although when a finger was not possible to evaluate additional pictures were taken on other fingers. Patients with fewer than eight images were excluded from the study. Quantitative and qualitative analyses of the images were performed blind to clinical data by investigator AS-O. Inter-observer reliability and intra-observer reliability were also explored with a calculated sample size of 45 patients. This sample was randomly selected and blindly assessed once by investigator AG-DC to calculate the inter-observer reproducibility. Afterwards, to estimate the intra-observer repeatability, a second round was independently performed by investigators AS-O and AG-DC with a minimal time interval of 2 weeks between both measurements.

Quantitative NVC was carried out according to previous definitions as follows: capillary density, number of capillaries measured in the distal row following the $90^{\circ}$ method [27]; enlarged capillary, an increase in capillary diameter $>20 \mu \mathrm{m}$; giant capillary, a capillary dilatation > $50 \mu \mathrm{m}$; microhaemorrhage, distal haemosiderin deposits; tortuous, curled capillaries with no cross; and neoangiogenesis, branching, bizarre, bushy, disorganized, ramified or arborized capillaries (Fig. 1) [14, 28, 29].

The mean of each capillaroscopic feature was calculated from the sum of consecutive images for each digit. Subsequently, the average values from eight fingers were added together and divided by the number of studied digits. The resulting value indicated the number of this capillaroscopic feature adjusted by each millimetre of the nailfold.

Qualitative NVC analysis of images was performed following capillaroscopy patterns described by Cutolo et al. (normal, early, active and late) [14].

\section{Statistical analysis}

For the descriptive statistics, qualitative data were expressed as the mean and standard deviation (SD) after approving the normal distribution test; and non-normal qualitative variables were described as the median and interquartile range (IQR). Normal distribution was assessed using the Kolmogorov-Smirnov test. To assess whether there were statistically significant differences between groups of patients, the Mann-Whitney $U$ test was used. Bonferroni correction was applied in multiple comparisons. Associations between NVC and clinical features, PFTs and echocardiography variables were analysed using Spearman's correlation.

In order to analyse inter-observer and intra-observer reliability, a sample size of 45 patients was calculated, based on two observers (AS-O and AG-DC), with a power of $80 \%$ to detect that an intra-class coefficient (ICC) of 0.7 was significantly higher than $0.45[30,31]$. The value of 0.45 was arbitrarily selected as the lowest acceptable limit of reliability. Quantitative NVC features were evaluated as the number per millimetre and determined by the ICC, whereas qualitative NVC features were assessed by Cohen's kappa coefficient. The measurement of observer agreement was defined as described in the literature: $<0.00=$ poor; $0.00-0.20=$ slight; $0.21-$ $0.40=$ fair; $0.41-0.60=$ moderate; $0.61-0.80=$ substantial; and $0.81-1.00=$ almost perfect [32] .

Multiple linear regression tests were performed to determine the association with FVC and DLCO. The multivariate models for FVC included NVC features, age at NVC, time elapsed from first symptom to NVC, dcSSc subset, gender, interstitial findings on HRCT, different autoantibodies and DU. For DLCO models, FVC, PAH confirmed by RHC, TRV $\geq 2.9 \mathrm{~m} / \mathrm{s}$ or other echo PH signs also were introduced. $p<0.05$ was considered significant. Statistical analysis was conducted with SPSS 20.0 for Windows (SPSS Inc., Chicago, IL, USA).

\section{Results \\ Population characteristics}

Demographic characteristics of the 134 SSc patients are presented in Table 1 . The participants were mainly female $(n=113,84.3 \%)$ and in the lcSSc subset $(n=88,65.7 \%)$. More than $90 \%$ of the population fulfilled the 2013 ACR/ EULAR classification criteria. ACA were the most common antibodies in 49 (36.6\%) patients, followed by anti-topoisomerase I antibodies in $31(23.1 \%)$ patients. Considering major organ involvement, digestive involvement was present in $110(82.1 \%)$ patients, cardiac disease in $103(76.9 \%)$ patients, ILD in $58(43.3 \%)$ patients, musculoskeletal involvement in $40(29.9 \%)$ patients and $\mathrm{PAH}$ in $11(8.2 \%)$ patients.

The distribution of different cardiac manifestations was $76.9 \%$ of patients presenting cardiac conduction 


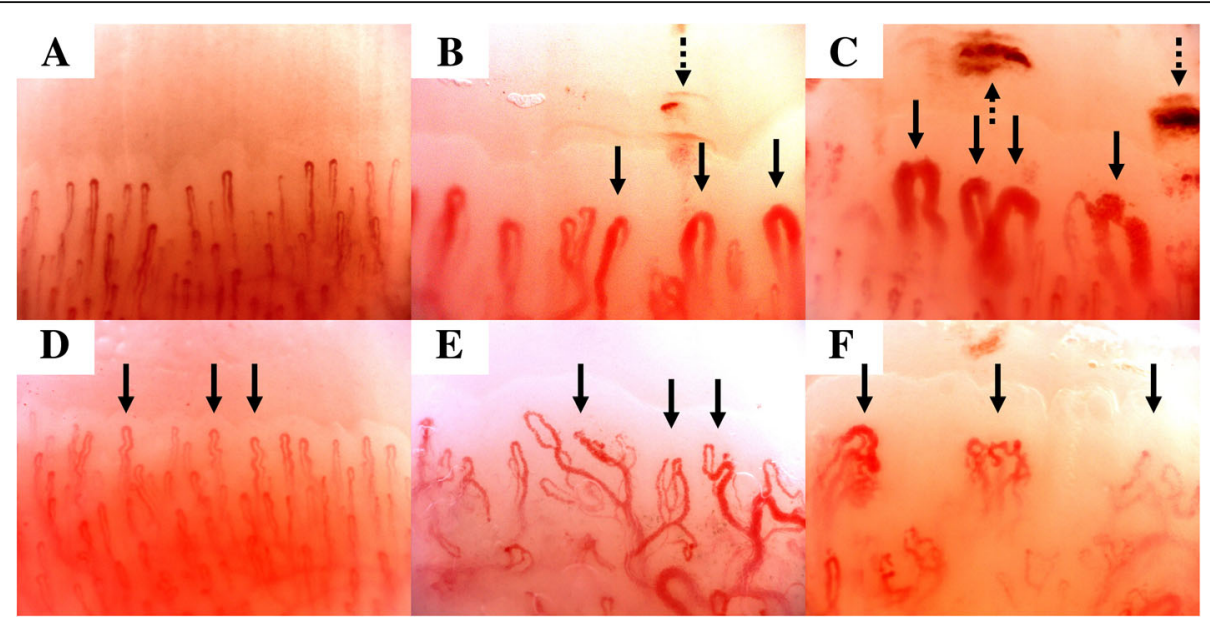

Fig. 1 Quantitative nailfold videocapillaroscopy: a normal capillaries; b enlarged capillaries (solid arrows) and microhaemorrhage (dashed arrow); c giant capillaries (solid arrows) and microhaemorrhages (dashed arrows); $\mathbf{d}$ tortuous capillaries (solid arrows); e capillaries with neoangiogenesis as ramified capillaries (solid arrows); $\mathbf{f}$ capillaries with neoangiogenesis as disorganized capillaries (solid arrows)

Table 1 Demographic, clinical and immunological characteristics of the patients with SSC $(n=134,100 \%)$

\begin{tabular}{lc}
\hline Variable & Result \\
\hline Age at SSc onset (years) & $38.5( \pm 15.7)$ \\
Age at NVC (years) & $54.7( \pm 14.4)$ \\
Cutaneous subsets, dcSSc/lcSSc/ssSSC & $28(20.9) / 88(65.7) / 18$ \\
& $(13.4)$ \\
Time elapsed from first symptom to & $16.0( \pm 12.6)$ \\
NVC (years) & $10.9( \pm 9.9)$ \\
Time elapsed from first non-RP symptom & \\
to NVC (years) & $121(90.2)$ \\
2013 ACR/EULAR SSc classification criteria & \\
fulfilment & $(36.6) / 31(23.1) / 14$ \\
ACA/ATA/anti-RNA pol III/anti-PM/Scl & $(10.4) / 11(8.2)$ \\
Raynaud's phenomenon & $132(98.5)$ \\
Telangiectasias & $97(72.4)$ \\
Digital ulcers & $62(46.3)$ \\
Calcinosis & $29(21.6)$ \\
Scleroderma renal crisis & $2(1.5)$ \\
Interstitial lung disease & $58(43.3)$ \\
Pulmonary arterial hypertension & $11(8.2)$ \\
Cardiac involvement & $103(76.9)$ \\
Musculoskeletal disease & $40(29.9)$ \\
Gastrointestinal involvement & $110(82.1)$ \\
Overlap features & $19(14.1)$ \\
\hline Data presed &
\end{tabular}

Data presented as mean ( \pm standard deviation) for continuous variables and as number (\%) for categorical variables

SSC systemic sclerosis, NVC nailfold videocapillaroscopy, dc SSC diffuse cutaneous systemic sclerosis, ICSSC limited cutaneous systemic sclerosis, ssSSC sine scleroderma systemic sclerosis, RP Raynaud's phenomenon, $A C R$ American College of Rheumatology, EULAR European League Against Rheumatism, ACA anticentromere antibodies, ATA anti-topoisomerase I abnormalities, $57.5 \%$ mitral regurgitation (with no CVRF), 47\% diastolic dysfunction (with no CVRF), $13.4 \%$ pericardial effusion, $8.2 \%$ microvascular ischemic heart disease (with no CVRF), 3.7\% LVEF < 50\% and 3\% of patients macrovascular ischemic heart disease (with no CVRF). Among all patients, there were 19 (14.1\%) with overlap features as follows: nine (6.7\%) patients had dermatomyositis, seven (5.2\%) patients had Sjögren syndrome and the other three (2.2\%) patients had rheumatoid arthritis.

\section{Pulmonary assessments}

PFTs and echocardiography findings are presented in Table 2. The mean predicted FVC $( \pm$ SD) was $80.8 \%( \pm$ $20.1)$, with a mean predicted DLCO of $66.2 \%( \pm 23.7)$. Regarding echocardiography, the TRV could be measured in 96 patients with a mean of $2.8 \mathrm{~m} / \mathrm{s}( \pm 0.3)$, and no other signs of $\mathrm{PH}$ were found in the remaining $38 \mathrm{pa}-$ tients. Among 14 (10.4\%) subjects with TRV higher than $2.9 \mathrm{~m} / \mathrm{s}$ and/or with other echo $\mathrm{PH}$ signs, seven patients had been previously diagnosed with PAH by RHC. On the other hand, only 4 out of 120 patients with TRV lower than $2.9 \mathrm{~m} / \mathrm{s}$ and no other echo $\mathrm{PH}$ signs had been formerly diagnosed with PAH by RHC.

\section{NVC characteristics}

A total of 2186 images were analysed from 134 patients, with a mean of $16.3( \pm 5.0)$ images per subject and the median (IQR) of images for each finger was $2(1-3)$. The median (IQR) of capillary density adjusted by each millimetre of nailfold was $5.44 / \mathrm{mm}$ (4.33-6.74) (Table 3). NVC findings according to the presence of ILD were compared. Median capillary density in ILD patients was $4.86 / \mathrm{mm}$, significantly lower than patients with no ILD whose density was $5.88 / \mathrm{mm}(p=0.005)$. Moreover, the 
Table 2 Pulmonary function test and echocardiography within a 3-month period of nailfold videocapillaroscopy

\begin{tabular}{lcc}
\hline Variable & $N$ & Result \\
\hline Pulmonary function test & & \\
FVC, \% predicted & 134 & $80.8( \pm 20.1)$ \\
DLCO, \% predicted & 134 & $66.2( \pm 23.7)$ \\
FVC\%/DLCO\% & 134 & $1.5( \pm 0.4)$ \\
EChocardiography & & \\
RVSP $(\mathrm{mmHg})$ & 96 & $30.7( \pm 12.0)$ \\
TRV $(\mathrm{m} / \mathrm{s})$ & 96 & $2.8( \pm 0.3)$ \\
TRV $\geq 2.9 \mathrm{~m} / \mathrm{s}$ or other echo PH signs & 134 & $14(10.4)$ \\
\hline
\end{tabular}

Data presented as mean ( \pm standard deviation) for continuous variables and as number (\%) for categorical variables

FVC forced vital capacity, DLCO diffusion capacity of the lung for carbon monoxide, RVSP right ventricular systolic pressure, TRV tricuspid regurgitation velocity, $\mathrm{PH}$ pulmonary hypertension

number of capillaries with neoangiogenesis was higher in ILD patients $(0.56 / \mathrm{mm}$ vs $0.31 / \mathrm{mm}, p=0.005)$. Focusing on $\mathrm{PAH}$, patients with this manifestation also showed greater frequency of capillaries with neoangiogenesis compared with patients with no PAH $(0.70 / \mathrm{mm}$ vs $0.33 / \mathrm{mm}, p=0.007)$. All prior comparisons remained statistically significant after Bonferroni correction $(p<$ 0.008).

Concerning qualitative NVC analysis, a late pattern (27.6\%) was more prevalent in patients with ILD ( $p=$ 0.006). In addition, a late pattern showed lower FVC and DLCO values compared with normal, early and active patterns (Table 4).

\section{Clinical and NVC correlations}

Age at NVC was inversely correlated with the number of enlarged capillaries $(\rho=-0.20, p=0.01)$ and the quantity of microhaemorrhages $(\rho=-0.25, p=0.003)$. The time elapsed from first symptom to NVC was negatively associated with the number of microhaemorrhages $(\rho=-$ $0.25, p=0.003)$ and positively with the number of capillaries with neoangiogenesis $(\rho=0.18, p=0.03)$. Higher capillary density was positively correlated with the $\%$ DLCO $(\rho=0.26, p=0.003)$ and negatively with the RVSP $(\rho=-0.21, p=0.03)$. The number of capillaries with neoangiogenesis was inversely associated with \% FVC ( $\rho$ $=-0.24, p=0.004)$ and $\%$ DLCO $(\rho=-0.26, p=0.002)$ and was positively associated with $\operatorname{RVSP}(\rho=0.20, p=$ $0.04)$.

\section{Inter-observer and intra-observer reliability}

The inter-observer reproducibility for all quantitative NVC features was almost perfect with an ICC higher than 0.80 (Table 5), except for the number of capillaries with neoangiogenesis which displayed a substantial reproducibility $(95 \% \mathrm{CI})$ of $0.77(0.58-0.88)$. Qualitative Cutolo's patterns also showed almost perfect reproducibility with Cohen's $\kappa$ of $0.83(0.66-1.0)$, even when each pattern was analysed separately, with the only exception of an early pattern which had a substantial reproducibility with Cohen's $\kappa$ of $0.73(0.42-1.0)$.

Regarding intra-observer repeatability, similar results were obtained for quantitative and qualitative variables, with an increment of neoangiogenesis repeatability up to $0.81(0.56-0.90)$.

\section{Multivariate analysis}

Quantitative NVC studies To elucidate which NVC findings were more strongly associated with pulmonary parameters, a multivariate analysis was conducted using

Table 3 Nailfold videocapillaroscopy characteristics and comparisons between groups of patients depending on pulmonary involvement

\begin{tabular}{|c|c|c|c|c|c|c|}
\hline & \multicolumn{2}{|c|}{ ILD comparisons } & \multirow[t]{2}{*}{$p$ value } & \multicolumn{2}{|c|}{ PAH comparisons } & \multirow[t]{2}{*}{$p$ value } \\
\hline & ILD $(n=58)$ & No ILD $(n=76)$ & & $\mathrm{PAH}(n=11)$ & No PAH $(n=123)$ & \\
\hline \multicolumn{7}{|l|}{ Quantitative NVC features } \\
\hline Capillary density $(n)^{a}$ & $4.86(4.14-5.80)$ & $5.88(4.74-6.85)$ & $0.005^{*}$ & $5.47(5.00-5.83)$ & $5.42(4.31-6.74)$ & 0.997 \\
\hline Enlarged capillaries $(n)^{\text {a }}$ & $0.84(0.31-1.53)$ & $0.85(0.39-1.58)$ & 0.738 & $0.56(0.25-0.93)$ & $0.91(0.40-1.61)$ & 0.128 \\
\hline Giant capillaries $(n)$ a & $0.26(0.00-0.64)$ & $0.30(0.04-1.07)$ & 0.362 & $0.06(0.00-0.40)$ & $0.29(0.03-1.02)$ & 0.167 \\
\hline Microhaemorrhages $(n)^{\text {a }}$ & $0.12(0.00-0.38)$ & $0.13(0.00-0.42)$ & 0.735 & $0.06(0.00-0.22)$ & $0.14(0.00-0.40)$ & 0.101 \\
\hline Tortuous capillaries $(n)^{a}$ & $0.45(0.19-1.31)$ & $0.72(0.28-1.49)$ & 0.216 & $0.63(0.25-1.71)$ & $0.59(0.23-1.43)$ & 0.773 \\
\hline Neoangiogenesis $(n)^{\text {a }}$ & $0.56(0.23-1.28)$ & $0.31(0.12-0.62)$ & $0.005^{*}$ & $0.70(0.47-1.80)$ & $0.33(0.12-0.78)$ & $0.007^{*}$ \\
\hline \multicolumn{7}{|l|}{ Qualitative NVC features } \\
\hline $\begin{array}{l}\text { Cutolo's pattern, early/ } \\
\text { active/late }\end{array}$ & $\begin{array}{c}8(13.8) / 23(39.7) / 23 \\
(39.7)\end{array}$ & $\begin{array}{c}15(19.7) / 36(47.4) / 14 \\
(18.4)\end{array}$ & $\begin{array}{c}0.366 / 0.373 / \\
0.006^{*}\end{array}$ & $\begin{array}{c}1(9.1) / 4(36.4) / 6 \\
(54.5)\end{array}$ & $\begin{array}{c}21(17.9) / 52(44.4) / 29 \\
(24.8)\end{array}$ & $\begin{array}{c}0.688 / 0.755 / \\
0.069\end{array}$ \\
\hline
\end{tabular}

Data presented as median (interquartile range) for continuous variables and as number (\%) for categorical variables

ILD interstitial lung disease, PAH pulmonary arterial hypertension, NVC nailfold videocapillaroscopy

aAdjusted by each millimetre of the nailfold

* Statistically significant comparison after Bonferroni correction $(p<0.008)$ 
Table 4 Association between qualitative nailfold videocapillaroscopy and pulmonary function tests

\begin{tabular}{lccccc}
\hline & \multicolumn{4}{c}{ Cutolo's pattern } \\
\cline { 2 - 5 } & Normal $(n=15)$ & Early $(n=23)$ & Active $(n=59)$ & Late $(n=37)$ & $p$ value \\
\hline FVC, \% predicted & $89.1( \pm 20.3)$ & $82.3( \pm 17.2)$ & $85.3( \pm 18.3)$ & $69.6( \pm 20.7)^{*, * * *}$ \\
DLCO, \% predicted & $77.3( \pm 20.8)$ & $71.4( \pm 27.5)$ & $68.6( \pm 20.4)$ & $54.2( \pm 23.3)^{*,+, \neq}$ & 0.001 \\
\hline
\end{tabular}

Data presented as mean ( \pm standard deviation) for continuous variables

FVC forced vital capacity, DLCO diffusion capacity of the lung for carbon monoxide

${ }^{*} p<0.01$, late versus normal

${ }^{* *} p=0.001$, late versus active

${ }^{+} p<0.05$, late versus early

${ }^{\ddagger} p<0.05$, late versus active

\% FVC as a dependent variable. Table 6 presents variables with statistical significance or a tendency to be significant. The number of capillaries with neoangiogenesis in NVC, male gender and ILD on HRCT were associated with a lower \% FVC, whereas ACA positivity was identified as a protective factor. The analysis estimated that for each capillary with neoangiogenesis visualized on average per 1-mm nailfold, the FVC would be reduced $7.3 \%$.

A lower \% DLCO value was associated with a reduced number of enlarged capillaries and an increased number of giant capillaries, along with ILD on HRCT, lower \% FVC, PAH confirmed by RHC, TRV $\geq 2.9 \mathrm{~m} / \mathrm{s}$ or other echo $\mathrm{PH}$ signs on echocardiography. In this way, for each enlarged capillary on average per $1 \mathrm{~mm}$ the DLCO would be $7.8 \%$ higher; however, for each giant capillary it would be diminished $11.8 \%$.

Qualitative NVC studies Multivariate analysis showed an association between late pattern and lower \% FVC values. FVC would be diminished $14.3 \%$ in a patient with late NVC pattern features (Table 7). None of the NVC patterns were related to DLCO values.

Table 5 Inter-observer and intra-observer reliability

\begin{tabular}{lcc}
\hline & Inter-observer $(95 \% \mathrm{Cl})$ & Intra-observer (95\% Cl) \\
\hline ICC for quantitative NVC features & \\
Capillary density & $0.96(0.93-0.98)$ & $0.97(0.93-0.98)$ \\
Enlarged capillaries & $0.87(0.77-0.93)$ & $0.93(0.83-0.97)$ \\
Giant capillaries & $0.91(0.83-0.95)$ & $0.95(0.90-0.97)$ \\
Microhaemorrhages & $0.97(0.94-0.98)$ & $0.99(0.98-0.99)$ \\
Tortuous capillaries & $0.92(0.85-0.95)$ & $0.91(0.69-0.96)$ \\
Neoangiogenesis & $0.77(0.58-0.88)$ & $0.81(0.56-0.90)$ \\
Cohen's K coefficient for qualitative NVC features & \\
Cutolo's patterns & $0.83(0.66-1.0)$ & $0.81(0.64-0.97)$ \\
Normal pattern & $0.92(0.75-1.0)$ & $0.83(0.55-1.0)$ \\
Early pattern & $0.73(0.42-1.0)$ & $0.66(0.33-0.99)$ \\
Active pattern & $0.82(0.65-0.99)$ & $0.87(0.71-1.0)$ \\
Late pattern & $0.87(0.69-1.0)$ & $0.81(0.59-10)$ \\
\hline
\end{tabular}

$\mathrm{Cl}$ confidence interval, ICC intra-class coefficient, NVC nailfold videocapillaroscopy

\section{Discussion}

This cross-sectional study in an SSc population indicated that the presence of capillaries with neoangiogenesis in NVC was related to lower percentage FVC values independently of other baseline characteristics. According to our results, a patient with one capillary with neoangiogenesis per millimetre on average in NVC would have $7.3 \%$ lower FVC percentage regardless of other clinical or capillaroscopic conditions. Concerning qualitative NVC assessments, a late pattern was also associated with lower FVC. We also found a substantial or almost perfect inter-observer and intra-observer reliability of all NVC features.

The median capillary density was similar to that reported previously, but our study included a higher significant number of evaluated images per patient [33, 34]. Prior publications have mainly focused on qualitative $\mathrm{NVC}$, finding correlations between NVC patterns and different variables such as ILD, FVC, DLCO, RVSP, DU

Table 6 Multiple regression analysis according to quantitative nailfold videocapillaroscopy

\begin{tabular}{lccc}
\hline & $B$ & $95 \% \mathrm{Cl}$ & $p$ value \\
\hline FVC as dependent variable & & & \\
Male gender & -11.0 & -19.2 to -2.9 & 0.008 \\
Interstitial findings on HRCT & -14.8 & -21.1 to -8.4 & $<0.001$ \\
ACA positivity & 7.2 & 0.1 to 14.3 & 0.045 \\
Neoangiogenesis/mm & -7.3 & -11.0 to -3.6 & $<0.001$ \\
DLCO as dependent variable & & & \\
Digital ulcers & -6.7 & -13.6 to 0.1 & 0.056 \\
Interstitial findings on HRCT & -9.4 & -16.4 to -2.5 & 0.008 \\
FVC & 0.5 & 0.3 to 0.7 & $<0.001$ \\
PAH confirmed by RHC & -14.3 & -26.9 to -1.6 & 0.027 \\
TRV $\geq 2.9$ m/s or other echo PH signs & -16.4 & -28.3 to -4.6 & 0.007 \\
Enlarged capillaries & 7.8 & 1.1 to 14.4 & 0.021 \\
Giant capillaries & -11.8 & -21.4 to -2.2 & 0.016 \\
\hline
\end{tabular}

Only variables with statistical significance or tendency to be significant are presented

B regression coefficient, $\mathrm{Cl}$ confidence interval, FVC forced vital capacity, HRCT high resolution computed tomography, ACA anticentromere antibodies, DLCO diffusion capacity of the lung for carbon monoxide, $P A H$ pulmonary arterial hypertension, $R H C$ right heart catheterization, $T R V$ tricuspid regurgitation velocity, $P H$ pulmonary hypertension 
Table 7 Multiple regression analysis according to Cutolo's patterns

\begin{tabular}{lccc}
\hline & $B$ & $95 \% \mathrm{Cl}$ & $p$ value \\
\hline FVC as dependent variable & & & \\
Male gender & -12.3 & -21.0 to -3.5 & 0.006 \\
Interstitial findings on HRCT & -13.5 & -20.2 to -6.7 & $<0.001$ \\
ACA positivity & 8.6 & -0.1 to 17.4 & 0.051 \\
Cutolo's late pattern & -14.3 & -26.1 to -2.5 & 0.018 \\
DLCO as dependent variable & & & \\
Digital ulcers & -8.3 & -14.8 to -1.8 & 0.013 \\
Interstitial findings on HRCT & -9.8 & -16.8 to -2.8 & 0.006 \\
FVC & 0.5 & 0.3 to 0.7 & $<0.001$ \\
PH confirmed by RHC & -14.1 & -26.8 to -1.5 & 0.028 \\
TRV $\geq 2.9$ m/s or other echo PH signs & -14.9 & -26.8 to -3.0 & 0.014 \\
\hline
\end{tabular}

Only variables with statistical significance or tendency to be significant are presented

$\mathrm{B}$ regression coefficient, $\mathrm{Cl}$ confidence interval, $F V C$ forced vital capacity, HRCT high resolution computed tomography, $A C A$ anticentromere antibodies, DLCO diffusion capacity of the lung for carbon monoxide, $P A H$ pulmonary arterial hypertension, $\mathrm{PH}$ pulmonary hypertension, $R H C$ right heart catheterization, $T R V$ tricuspid regurgitation velocity

or even risk of developing new DU [35-38]. On the other hand, quantitative NVC studies proposed capillaroscopic prognostic tools for the development of new DU or even the progression to SSc [34, 39-41]. We achieved equal or superior intra-observer and inter-observer reliability to previous quantitative NVC studies [27], probably because our work compared the mean of NVC features analysing all images of each patient instead of a single image per patient, which reflects real-life capillaroscopy more. Moreover, qualitative NVC features as Cutolo's patterns were assessed with almost perfect reliability. However, few research projects have concentrated on extensive quantitative analysis and its correlation with clinical variables, specifically with lung parameters.

We found that SSc patients with ILD had a lower capillary density and a higher number of capillaries with neoangiogenesis. These findings support the prior semi-quantitative NVC study, which identified higher mean avascular scores in patients with ground-glass opacities [42]. Similarly, Castellvi et al. [43] described an association between low capillary density defined as $<7$ capillaries/mm and lower FVC and DLCO percentages using a semi-quantitative NVC. Smith et al. [37] also found a strong association between NVC patterns and future severe lung involvement at 18-24 months, defined as a punctuation $\geq 2$ in lung evaluation on the Medsger severity scale. Nevertheless, the risk of severe ILD or PH was not specified separately [37, 44].

Recently, Avouac et al. [16] published a sequential quantitative NVC study with multivariate Cox analysis revealing that an incident or increased number of giant capillaries during the follow-up was protective of new DU. Progressive loss of capillaries from baseline predicted overall disease progression, new DU, lung vascular progression defined as new onset of precapillary $\mathrm{PH}$ on RHC, skin fibrosis and worsening in the Medsger severity score. Baseline angiogenesis predicted only lung vascular progression. The present study performs a multivariate analysis including clinical, immunological and quantitative/qualitative NVC findings for the investigation of lung parameters at baseline conditions. We identified that the number of capillaries with neoangiogenesis, male gender and the presence of ILD on HRCT were factors independently associated with lower FVC values, while ACA positivity was found to be a protector, which supports Avouac et al.'s work.

Furthermore, regarding qualitative NVC studies, the progression from normal/early/active to a late pattern was related to the development of new DU, lung vascular progression, progression of skin fibrosis and worsening on the Medsger severity scale [16]. According to these findings, the greatest destructive NVC features, as the late pattern, were associated with lower FVC values.

Consequently, it appears that endothelial damage mediates both lung fibrosis and peripheral microvascular changes visualized in NVC. SSc patients with more extensive ILD may also show higher nailfold capillary perturbations, ineffective angiogenesis and vasculogenesis, which reflects a higher frequency of bushy or bizarre capillaries which are denominated as neoangiogenesis [45]. In fact, ILD has already been linked in a multivariate analysis with peripheral vasculopathy in the form of prior/current DU [20].

Recently, a qualitative NVC study has noted the predictive role of NVC and specific autoantibodies in cardiopulmonary involvement [35]. Specifically, active and late NVC patterns were associated with ILD, DLCO $<70 \%$ of predicted, reduced maximum oxygen uptake, higher NT pro-BNP and increased systolic pulmonary artery pressure values. However, $\mathrm{PH}$ was estimated by indirect measurements and was not confirmed by RHC. In our cohort, the prevalence of PAH confirmed by RHC was $8.2 \%$, similar to previous reports $[7,8]$. Surprisingly, we found enlarged capillaries being associated with higher \% DLCO values, and giant capillaries were independently correlated with lower DLCO percentage. These findings contrast with a prior publication where new giant or increased giant capillaries during follow-up tended to be protective of lung vascular progression or overall disease progression [16]. This paradoxical association between enlarged capillaries and higher DLCO values could be explained as an adaptive phenomenon. When enlarged capillaries turn into giant capillaries, which means a failure in microvascular adaptation and consequently a severe capillary dysfunction, an impairment of DLCO values occurs. The formation of extremely large capillaries may contribute to gas 
exchange disturbance in all tissues including the lung, which might result in DLCO decline.

Hofstee et al. [46] found that capillary density was inversely correlated with the MPAP in both SSc-PAH and idiopathic PAH patients. Interestingly, Riccieri et al. [47] demonstrated the existence of greater avascular areas and severe active/late NVC patterns in a group of 12 SSc-PAH patients, and that higher NVC scores and avascular areas scores were correlated with mPAP. Corrado et al. [48] observed capillaroscopic abnormalities in $38.1 \%$ of idiopathic PAH patients, with lower capillary density and higher loop width compared to healthy subjects. Furthermore, the authors confirmed a reduced capillary density, and an increased mean capillary width and mean number of capillaries with neoangiogenesis in SSc-PAH patients compared to SSc patients without $\mathrm{PAH}$ evidence. Although we also identified a negative correlation of higher capillary density and number of neoangiogenesis capillaries with RVSP values, we did not found an association between those NVC features and PAH defined by RHC in the multivariate analysis, which may be due to the small group of PAH patients.

Limitations of this study included the cross-sectional study, with no follow-up data, which did not allow us to infer whether capillaroscopic findings were linked to a specific outcome of pulmonary disease. However, although an association was observed between microvascular features and functional respiratory parameters, this fact does not prove a causal relationship between them. We cannot exclude that both phenomena would be influenced by an unknown parameter that may lead a parallel change in the first two. Notwithstanding, the present study also had strengths, because all patients had undergone echocardiography and PFTs close to the NVC examination. The images were analysed blind of clinical data by the researchers. For the statistical analysis, patients were excluded with fewer than eight explored fields, either due to technical difficulties or digital amputations. NVC explorations after autologous stem cell transplantation had demonstrated an improvement in vascular damage [49]. Consequently, five lung transplant patients under high immunosuppressive treatment were additionally removed from the study.

\section{Conclusions}

This study describes a lower capillary density and a higher number of capillaries with neoangiogenesis in patients with ILD, and demonstrates a quantitative and qualitative relation between specific NVC abnormalities and lung function tests (both \% FVC and \% DLCO parameters). It seems that NVC findings are linked to a wide spectrum of clinical variables in SSc, which emphasizes the crucial role of microcirculation damage in this medical condition, especially within the lung.

\section{Abbreviations}

ACA: Anticentromere antibodies; ANA: Antinuclear antibodies; Cl: Confidence interval; CVRF: Cardiovascular risk factor; dcSSc: Diffuse cutaneous SSc; DLCO: Diffusion capacity of the lung for carbon monoxide; DU: Digital ulcers; FVC: Forced vital capacity; HRCT: High-resolution computed tomography; ICC: Intra-class coefficient; IIF: Indirect immunofluorescence; ILD: Interstitial lung disease; IQR: Interquartile range; IcSSc: Limited cutaneous SSC; LVEF: Left ventricular ejection fraction; mPAP: Mean pulmonary arterial pressure; NVC: Nailfold videocapillaroscopy; PAH: Pulmonary arterial hypertension; PAWP: Pulmonary artery wedge pressure; PFT: Pulmonary function test; PH: Pulmonary hypertension; PVR: Pulmonary vascular resistance; RHC: Right heart catheterization; RP: Raynaud's phenomenon; RVSP: Right ventricular systolic pressure; SD: Standard deviation; SRC: Scleroderma renal crisis; SSc: Systemic sclerosis; ssSSc: Sine scleroderma SSc; STROBE: Strengthening the Reporting of Observational Studies in Epidemiology; TRV: Tricuspid regurgitation velocity; VEGF: Vascular endothelial growth factor

\section{Acknowledgements}

Not applicable.

\section{Funding}

This work was supported by the Instituto de Salud Carlos III and the European Regional Development Fund (ERDF) (grant numbers PI15/02100 and PI16/02088).

\section{Availability of data and materials}

The datasets used and/or analysed during the current study are available from the corresponding author on reasonable request.

\section{Authors' contributions}

AG-DC made substantial contributions to study conception and design, as well as acquisition, analysis and interpretation of data, drafting the article and approval of the final manuscript. CPS-A made substantial contributions to study conception and design, analysis and interpretation of data, revising the manuscript critically for important intellectual content and approval of the final manuscript. ELC-M, CT-V, SA-V and VF-P made substantial contributions to study conception and design, revising the manuscript critically for important intellectual content and approval of the final manuscript. AS-O made substantial contributions to study conception and design, acquisition, analysis and interpretation of data, revising the manuscript critically for important intellectual content and approval of the final manuscript. AG-DC and CPS-A had full access to all of the data in the study and both take responsibility for the integrity and accuracy of the data. Funders had no access to the data and were not involved in study design, acquisition, analysis and interpretation of data or writing the manuscript.

\section{Ethics approval and consent to participate}

The study was approved by the Ethics Committee for Clinical Research (PG(AG)4/2015), and all patients provided written informed consent for their participation.

\section{Consent for publication}

Not applicable.

\section{Competing interests}

The authors declare that they have no competing interests.

\section{Publisher's Note}

Springer Nature remains neutral with regard to jurisdictional claims in published maps and institutional affiliations.

\section{Author details}

'Department of Systemic Autoimmune Diseases, Hospital Universitari Vall d'Hebron, Universitat Autònoma de Barcelona, Passeig Vall d'Hebron 119129, 08035 Barcelona, Spain. ²Department of Internal Medicine, Corporació Sanitària Universitària Parc Taulí, Universitat Autònoma de Barcelona, Sabadell, Barcelona, Spain. 
Received: 28 May 2018 Accepted: 25 November 2018 Published online: 19 December 2018

\section{References}

1. Denton CP, Ong VH. Targeted therapies for systemic sclerosis. Nat Rev Rheumatol. 2013;9:451-64.

2. Allanore $Y$, Simms R, Distler O, Trojanowska M, Pope J, Denton CP, et al. Systemic sclerosis. Nat Rev Dis Primers. 2015;1:15002.

3. Denton CP, Khanna D. Systemic sclerosis. Lancet. 2017:390:1685-99.

4. Bhattacharyya S, Wei J, Varga J. Understanding fibrosis in systemic sclerosis: shifting paradigms, emerging opportunities. Nat Rev Rheumatol. 2011:8:42-54.

5. Launay D, Remy-Jardin M, Michon-Pasturel U, Mastora I, Hachulla E, Lambert $M$, et al. High resolution computed tomography in fibrosing alveolitis associated with systemic sclerosis. J Rheumatol. 2006:33:1789-801.

6. De Santis M, Bosello S, La Torre G, Capuano A, Tolusso B, Pagliari G, et al. Functional, radiological and biological markers of alveolitis and infections of the lower respiratory tract in patients with systemic sclerosis. Respir Res. 2005;6:96.

7. Mukerjee D, St George D, Coleiro B, Knight C, Denton CP, Davar J, et al. Prevalence and outcome in systemic sclerosis associated pulmonary arterial hypertension: application of a registry approach. Ann Rheum Dis. 2003;62: 1088-93.

8. Avouac J, Airo P, Meune C, Beretta L, Dieude P, Caramaschi P, et al. Prevalence of pulmonary hypertension in systemic sclerosis in European Caucasians and metaanalysis of 5 studies. J Rheumatol. 2010:37:2290-8.

9. Steen VD, Medsger TA. Changes in causes of death in systemic sclerosis, 1972-2002. Ann Rheum Dis. 2007:66:940-4.

10. Nihtyanova SI, Schreiber BE, Ong VH, Rosenberg D, Moinzadeh P, Coghlan $J G$, et al. Prediction of pulmonary complications and long-term survival in systemic sclerosis. Arthritis Rheumatol. 2014;66:1625-35.

11. Simeon-Aznar CP, Fonollosa-Pla V, Tolosa-Vilella C, Espinosa-Garriga G, Campillo-Grau M, Ramos-Casals M, et al. Registry of the Spanish Network for Systemic Sclerosis: Survival, Prognostic Factors, and Causes of Death. Medicine (Baltimore). 2015:94:e1728

12. Maricq HR, LeRoy EC, D'Angelo WA, Medsger TA Jr, Rodnan GP, Sharp GC, et al. Diagnostic potential of in vivo capillary microscopy in scleroderma and related disorders. Arthritis Rheum. 1980;23:183-9.

13. Marica HR, Harper FE, Khan MM, Tan EM, LeRoy EC. Microvascular abnormalities as possible predictors of disease subsets in Raynaud phenomenon and early connective tissue disease. Clin Exp Rheumatol. 1983:1:195-205

14. Cutolo M, Sulli A, Pizzorni C, Accardo S. Nailfold videocapillaroscopy assessment of microvascular damage in systemic sclerosis. J Rheumatol. 2000;27:155-60.

15. Avouac J, Vallucci M, Smith V, Senet P, Ruiz B, Sulli A, et al. Correlations between angiogenic factors and capillaroscopic patterns in systemic sclerosis. Arthritis Res Ther. 2013;15:R55.

16. Avouac J, Lepri G, Smith V, Toniolo E, Hurabielle C, Vallet A, et al. Sequential nailfold videocapillaroscopy examinations have responsiveness to detect organ progression in systemic sclerosis. Semin Arthritis Rheum. 2017:47:86-94.

17. LeRoy EC, Black C, Fleischmajer R, Jablonska S, Krieg T, Medsger TA Jr, et al. Scleroderma (systemic sclerosis): classification, subsets and pathogenesis. J Rheumatol. 1988;15:202-5.

18. van den Hoogen F, Khanna D, Fransen J, Johnson SR, Baron M, Tyndall A, et al. 2013 classification criteria for systemic sclerosis: an American College of Rheumatology/European League Against Rheumatism collaborative initiative. Ann Rheum Dis. 2013:72:1747-55.

19. von Elm E, Altman DG, Egger M, Pocock SJ, Gotzsche PC, Vandenbroucke JP, et al. The Strengthening the Reporting of Observational Studies in Epidemiology (STROBE) statement: guidelines for reporting observationa studies. Epidemiology. 2007;18:800-4.

20. Tolosa-Vilella C, Morera-Morales ML, Simeon-Aznar CP, Mari-Alfonso B, Colunga-Arguelles D, Callejas Rubio JL, et al. Digital ulcers and cutaneous subsets of systemic sclerosis: clinical, immunological, nailfold capillaroscopy, and survival differences in the Spanish RESCLE Registry. Semin Arthritis Rheum. 2016:46:200-8.

21. Morales-Cardenas A, Perez-Madrid C, Arias L, Ojeda P, Mahecha MP, RojasVillarraga $A$, et al. Pulmonary involvement in systemic sclerosis. Autoimmun Rev. 2016;15:1094-108.
22. Coghlan JG, Denton CP, Grunig E, Bonderman D, Distler O, Khanna D, et al. Evidence-based detection of pulmonary arterial hypertension in systemic sclerosis: the DETECT study. Ann Rheum Dis. 2014;73:1340-9.

23. Galie N, Humbert M, Vachiery JL, Gibbs S, Lang I, Torbicki A, et al. 2015 ESC/ ERS Guidelines for the diagnosis and treatment of pulmonary hypertension: The Joint Task Force for the Diagnosis and Treatment of Pulmonary Hypertension of the European Society of Cardiology (ESC) and the European Respiratory Society (ERS): Endorsed by: Association for European Paediatric and Congenital Cardiology (AEPC), International Society for Heart and Lung Transplantation (ISHLT). Eur Heart J. 2016;37:67-119.

24. Fernandez-Codina A, Simeon-Aznar CP, Pinal-Fernandez I, RodriguezPalomares J, Pizzi MN, Hidalgo CE, et al. Cardiac involvement in systemic sclerosis: differences between clinical subsets and influence on survival. Rheumatol Int. 2017;37:75-84. https://doi.org/10.1007/s00296-015-3382-2.

25. Miller MR, Crapo R, Hankinson J, Brusasco V, Burgos F, Casaburi R, et al. General considerations for lung function testing. Eur Respir J. 2005;26: 153-61.

26. Lang RM, Badano LP, Mor-Avi V, Afilalo J, Armstrong A, Ernande L, et al. Recommendations for cardiac chamber quantification by echocardiography in adults: an update from the American Society of Echocardiography and the European Association of Cardiovascular Imaging. Eur Heart J Cardiovasc Imaging. 2015;16:233-70.

27. Hofstee HM, Serne EH, Roberts $C$, Hesselstrand $R$, Scheja A, Moore $T L$, et al. A multicentre study on the reliability of qualitative and quantitative nail-fold videocapillaroscopy assessment. Rheumatology (Oxford). 2012;51:749-55.

28. Sulli A, Secchi ME, Pizzorni C, Cutolo M. Scoring the nailfold microvascular changes during the capillaroscopic analysis in systemic sclerosis patients. Ann Rheum Dis. 2008:67:885-7.

29. Maricq HR. Wide-field capillary microscopy. Arthritis Rheum. 1981;24: 1159-65.

30. Donner A, Eliasziw M. Sample size requirements for reliability studies. Stat Med. 1987:6:441-8.

31. Walter SD, Eliasziw M, Donner A. Sample size and optimal designs for reliability studies. Stat Med. 1998;17:101-10.

32. Landis JR, Koch GG. The measurement of observer agreement for categorical data. Biometrics. 1977:33:159-74.

33. Morardet L, Avouac J, Sammour M, Baron M, Kahan A, Feydy A, et al. Late nailfold videocapillaroscopy pattern associated with hand calcinosis and acro-osteolysis in systemic sclerosis. Arthritis Care Res (Hoboken). 2016;68: 366-73.

34. Cutolo M, Herrick AL, Distler O, Becker MO, Beltran E, Carpentier P, et al. Nailfold videocapillaroscopic features and other clinical risk factors for digital ulcers in systemic sclerosis: a multicenter, prospective cohort study. Arthritis Rheumatol. 2016:68:2527-39.

35. Markusse IM, Meijs J, de Boer B, Bakker JA, Schippers HP, Schouffoer AA, et al. Predicting cardiopulmonary involvement in patients with systemic sclerosis: complementary value of nailfold videocapillaroscopy patterns and disease-specific autoantibodies. Rheumatology (Oxford). 2017;56:1081-88. https://doi.org/10.1093/rheumatology/kew402.

36. Caramaschi P, Canestrini S, Martinelli N, Volpe A, Pieropan S, Ferrari M, et al. Scleroderma patients nailfold videocapillaroscopic patterns are associated with disease subset and disease severity. Rheumatology (Oxford). 2007;46: $1566-9$

37. Smith V, Decuman S, Sulli A, Bonroy C, Piettte Y, Deschepper E, et al. Do worsening scleroderma capillaroscopic patterns predict future severe organ involvement? A pilot study. Ann Rheum Dis. 2012;71:1636-9.

38. Silva I, Teixeira A, Oliveira J, Almeida I, Almeida R, Aguas A, et al. Endothelial dysfunction and nailfold videocapillaroscopy pattern as predictors of digital ulcers in systemic sclerosis: a cohort study and review of the literature. Clin Rev Allergy Immunol. 2015;49:240-52

39. Sebastiani M, Manfredi A, Colaci M, D'Amico R, Malagoli V, Giuggioli D, et al Capillaroscopic skin ulcer risk index: a new prognostic tool for digital skin ulcer development in systemic sclerosis patients. Arthritis Rheum. 2009;61: 688-94.

40. Sebastiani M, Manfredi A, Vukatana G, Moscatelli S, Riato L, Bocci M, et al. Predictive role of capillaroscopic skin ulcer risk index in systemic sclerosis: a multicentre validation study. Ann Rheum Dis. 2012;71:67-70.

41. Trombetta AC, Smith V, Pizzorni C, Meroni M, Paolino S, Cariti C, et al. Quantitative alterations of capillary diameter have a predictive value for development of the capillaroscopic systemic sclerosis pattern. J Rheumatol. 2016:43:599-606. 
42. Bredemeier M, Xavier RM, Capobianco KG, Restelli VG, Rohde LE, Pinotti AF, et al. Nailfold capillary microscopy can suggest pulmonary disease activity in systemic sclerosis. J Rheumatol. 2004;31:286-94.

43. Castellvi I, Simeon-Aznar CP, Sarmiento M, Fortuna A, Mayos M, Geli C, et al. Association between nailfold capillaroscopy findings and pulmonary function tests in patients with systemic sclerosis. J Rheumatol. 2015:42:222-7.

44. Medsger TA Jt, Bombardieri S, Czirjak L, Scorza R, Della Rossa A, Bencivelli W. Assessment of disease severity and prognosis. Clin Exp Rheumatol. 2003;21:S42-6.

45. Lambova SN, Muller-Ladner U. Capillaroscopic pattern in systemic sclerosis - an association with dynamics of processes of angio- and vasculogenesis. Microvasc Res. 2010;80:534-9.

46. Hofstee HM, Vonk Noordegraaf A, Voskuyl AE, Dijkmans BA, Postmus PE, Smulders $Y M$, et al. Nailfold capillary density is associated with the presence and severity of pulmonary arterial hypertension in systemic sclerosis. Ann Rheum Dis. 2009:68:191-5.

47. Riccieri V, Vasile M, lannace N, Stefanantoni K, Sciarra I, Vizza CD, et al. Systemic sclerosis patients with and without pulmonary arterial hypertension: a nailfold capillaroscopy study. Rheumatology (Oxford). 2013; 52:1525-8.

48. Corrado A, Correale M, Mansueto N, Monaco I, Carriero A, Mele A, et al. Nailfold capillaroscopic changes in patients with idiopathic pulmonary arterial hypertension and systemic sclerosis-related pulmonary arterial hypertension. Microvasc Res. 2017;114:46-51.

49. Miniati I, Guiducci S, Conforti ML, Rogai V, Fiori G, Cinelli M, et al. Autologous stem cell transplantation improves microcirculation in systemic sclerosis. Ann Rheum Dis. 2009:68:94-8.

Ready to submit your research? Choose BMC and benefit from:

- fast, convenient online submission

- thorough peer review by experienced researchers in your field

- rapid publication on acceptance

- support for research data, including large and complex data types

- gold Open Access which fosters wider collaboration and increased citations

- maximum visibility for your research: over $100 \mathrm{M}$ website views per year

At $\mathrm{BMC}$, research is always in progress.

Learn more biomedcentral.com/submissions 